\title{
Sign-graded posets, unimodality of $W$-polynomials and the Charney-Davis Conjecture
}

\author{
Petter Brändén* \\ Chalmers University of Technology and Göteborg University \\ S-412 96 Göteborg, Sweden \\ branden@math. chalmers.se
}

Submitted: Jul 6, 2004; Accepted: Nov 6, 2004; Published: Nov 22, 2004

Mathematics Subject Classifications: 06A07, 05E99, 13F55

Dedicated to Richard Stanley on the occasion of his 60th birthday

\begin{abstract}
We generalize the notion of graded posets to what we call sign-graded (labeled) posets. We prove that the $W$-polynomial of a sign-graded poset is symmetric and unimodal. This extends a recent result of Reiner and Welker who proved it for graded posets by associating a simplicial polytopal sphere to each graded poset. By proving that the $W$-polynomials of sign-graded posets has the right sign at -1 , we are able to prove the Charney-Davis Conjecture for these spheres (whenever they are flag).
\end{abstract}

\section{Introduction and preliminaries}

Recently Reiner and Welker [10] proved that the $W$-polynomial of a graded poset (partially ordered set) $P$ has unimodal coefficients. They proved this by associating to $P$ a simplicial polytopal sphere, $\Delta_{e q}(P)$, whose $h$-polynomial is the $W$-polynomial of $P$, and invoking the $g$-theorem for simplicial polytopes (see $[15,16]$ ). Whenever this sphere is flag, i.e., its minimal non-faces all have cardinality two, they noted that the Neggers-Stanley Conjecture implies the Charney-Davis Conjecture for $\Delta_{e q}(P)$. In this paper we give a different proof of the unimodality of $W$-polynomials of graded posets, and we also prove the Charney-Davis Conjecture for $\Delta_{e q}(P)$ (whenever it is flag). We prove it by studying a family of labeled posets, which we call sign-graded posets, of which the class of graded naturally labeled posets is a sub-class.

*Part of this work was financed by the EC's IHRP Programme, within the Research Training Network "Algebraic Combinatorics in Europe", grant HPRN-CT-2001-00272, while the author was at Universitá di Roma "Tor Vergata", Rome, Italy. 
In this paper all posets will be finite and non-empty. For undefined terminology on posets we refer the reader to [13]. We denote the cardinality of a poset $P$ with the letter $p$. Let $P$ be a poset and let $\omega: P \rightarrow\{1,2, \ldots, p\}$ be a bijection. The pair $(P, \omega)$ is called a labeled poset. If $\omega$ is order-preserving then $(P, \omega)$ is said to be naturally labeled. A $(P, \omega)$-partition is a map $\sigma: P \rightarrow\{1,2,3, \ldots\}$ such that

- $\sigma$ is order reversing, that is, if $x \leq y$ then $\sigma(x) \geq \sigma(y)$,

- if $x<y$ and $\omega(x)>\omega(y)$ then $\sigma(x)>\sigma(y)$.

The theory of $(P, \omega)$-partitions was developed by Stanley in [14]. The number of $(P, \omega)$ partitions $\sigma$ with largest part at most $n$ is a polynomial of degree $p$ in $n$ called the order polynomial of $(P, \omega)$ and is denoted $\Omega(P, \omega ; n)$. The $W$-polynomial of $(P, \omega)$ is defined by

$$
\sum_{n \geq 0} \Omega(P, \omega ; n+1) t^{n}=\frac{W(P, \omega ; t)}{(1-t)^{p+1}} .
$$

The set, $\mathcal{L}(P, \omega)$, of permutations $\omega\left(x_{1}\right), \omega\left(x_{2}\right), \ldots, \omega\left(x_{p}\right)$ where $x_{1}, x_{2}, \ldots, x_{p}$ is a linear extension of $P$ is called the Jordan-Hölder set of $(P, \omega)$. A descent in a permutation $\pi=\pi_{1} \pi_{2} \cdots \pi_{p}$ is an index $1 \leq i \leq p-1$ such that $\pi_{i}>\pi_{i+1}$. The number of descents in $\pi$ is denoted $\operatorname{des}(\pi)$. A fundamental result in the theory of $(P, \omega)$-partitions, see [14], is that the $W$-polynomial can be written as

$$
W(P, \omega ; t)=\sum_{\pi \in \mathcal{L}(P, \omega)} t^{\operatorname{des}(\pi)}
$$

The Neggers-Stanley Conjecture is the following:

Conjecture 1.1 (Neggers-Stanley). Let $(P, \omega)$ be a labeled poset. Then $W(P, \omega ; t)$ has real zeros only.

This was first conjectured by Neggers [8] in 1978 for natural labelings and by Stanley in 1986 for arbitrary labelings. The conjecture has been proved for some special cases, see $[1,2,10,17]$ for the state of the art. If a polynomial has only real non-positive zeros then its coefficients form a unimodal sequence. For the $W$-polynomials of graded posets unimodality was first proved by Gasharov [7] whenever the rank is at most 2, and as mentioned by Reiner and Welker [10] for all graded posets.

For the relevant definitions concerning the topology behind the Charney-Davis Conjecture we refer the reader to $[3,10,16]$.

Conjecture 1.2 (Charney-Davis, [3]). Let $\Delta$ be a flag simplicial homology $(d-1)$ sphere, where $d$ is even. Then the h-vector, $h(\Delta, t)$, of $\Delta$ satisfies

$$
(-1)^{d / 2} h(\Delta,-1) \geq 0
$$


Recall that the $n$th Eulerian polynomial, $A_{n}(x)$, is the $W$-polynomial of an anti-chain of $n$ elements. The Eulerian polynomials can be written as

$$
A_{n}(x)=\sum_{i=0}^{\lfloor(n-1) / 2\rfloor} a_{n, i} x^{i}(1+x)^{n-1-2 i}
$$

where $a_{n, i}$ is a nonnegative integer for all $i$, see $[5,11]$. From this expansion we see immediately that $A_{n}(x)$ is symmetric and that the coefficients in the standard basis are unimodal. It also follows that $(-1)^{(n-1) / 2} A_{n}(-1) \geq 0$.

We will in Section 2 define a class of labeled poset whose members we call sign-graded posets. This class includes the class of naturally labeled graded posets. In Section 4 we show that the $W$-polynomial of a sign-graded poset $(P, \omega)$ of rank $r$ can be expanded, just as the Eulerian polynomial, as

$$
W(P, \omega ; t)=\sum_{i=0}^{\lfloor(p-r-1) / 2\rfloor} a_{i}(P, \omega) t^{i}(1+t)^{p-r-1-2 i},
$$

where $a_{i}(P, \omega)$ are nonnegative integers. Hence, symmetry and unimodality follow, and $W(P, \omega ; t)$ has the right sign at -1 . Consequently, whenever the associated sphere $\Delta_{e q}(P)$ of a graded poset $P$ is flag the Charney-Davis Conjecture holds for $\Delta_{e q}(P)$. We also note that all symmetric polynomials with non-positive zeros only, admit an expansion such as (1.2). Hence, that $W(P, \omega ; t)$ has such an expansion can be seen as further evidence for the Neggers-Stanley Conjecture. After the completion of the first version of this paper we were informed that $\mathrm{S}$. Gal [6] has conjectured that if $\Delta$ is flag simplicial homology $(d-1)$-sphere, then its $h$-vector admits an expansion

$$
h(\Delta, t)=\sum_{i=0}^{\lfloor d / 2\rfloor} a_{i}(\Delta) t^{i}(1+t)^{d-2 i}
$$

where $a_{i}(\Delta)$ are nonnegative integers. This would imply the Charney-Davis conjecture and (1.2) can be seen as further evidence for Gal's conjecture.

In [9] the Charney-Davis quantity of a graded naturally labeled poset $(P, \omega)$ of rank $r$ was defined to be $(-1)^{(p-1-r) / 2} W(P, \omega ;-1)$. In Section 5 we give a combinatorial interpretation of the Charney-Davis quantity as counting certain reverse alternating permutations. Finally in Section 7 we characterize sign-graded posets in terms of properties of order polynomials.

\section{Sign-graded posets}

Recall that a poset $P$ is graded if all maximal chains in $P$ have the same length. If $P$ is graded one may associate a rank function $\rho: P \rightarrow \mathbb{N}$ by letting $\rho(x)$ be the length of any saturated chain from a minimal element to $x$. The rank of a graded poset $P$ is defined 
Figure 1: A sign-graded poset, its two labelings and the corresponding rank function.
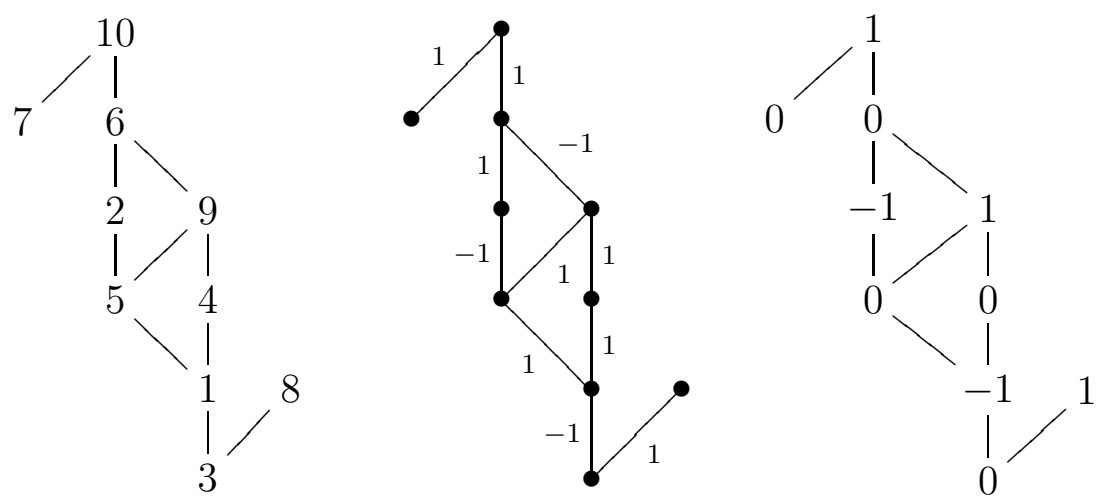

as the length of any maximal chain in $P$. In this section we will generalize the notion of graded posets to labeled posets.

Let $(P, \omega)$ be a labeled poset. An element $y$ covers $x$, written $x \prec y$, if $x<y$ and $x<z<y$ for no $z \in P$. Let $E=E(P)=\{(x, y) \in P \times P: x \prec y\}$ be the covering relations of $P$. We associate a labeling $\epsilon: E \rightarrow\{-1,1\}$ of the covering relations defined by

$$
\epsilon(x, y)=\left\{\begin{aligned}
1 \text { if } & \omega(x)<\omega(y) \\
-1 \text { if } & \omega(x)>\omega(y)
\end{aligned}\right.
$$

If two labelings $\omega$ and $\lambda$ of $P$ give rise to the same labeling of $E(P)$ then it is easy to see that the set of $(P, \omega)$-partitions and the set of $(P, \lambda)$-partitions are the same. In what follows we will often refer to $\epsilon$ as the labeling and write $(P, \epsilon)$.

Definition 2.1. Let $(P, \omega)$ be a labeled poset and let $\epsilon$ be the corresponding labeling of $E(P)$. We say that $(P, \omega)$ is sign-graded, and that $P$ is $\epsilon$-graded (and $\omega$-graded) if for every maximal chain $x_{0} \prec x_{1} \prec \cdots \prec x_{n}$ the sum

$$
\sum_{i=1}^{n} \epsilon\left(x_{i-1}, x_{i}\right)
$$

is the same. The common value of the above sum is called the rank of $(P, \omega)$ and is denoted $r(\epsilon)$.

We say that the poset $P$ is $\epsilon$-consistent (and $\omega$-consistent) if for every $y \in P$ the principal order ideal $\Lambda_{y}=\{x \in P: x \leq y\}$ is $\epsilon_{y}$-graded, where $\epsilon_{y}$ is $\epsilon$ restricted to $E\left(\Lambda_{y}\right)$. The rank function $\rho: P \rightarrow \mathbb{Z}$ of an $\epsilon$-consistent poset $P$ is defined by $\rho(x)=r\left(\epsilon_{x}\right)$. Hence, an $\epsilon$-consistent poset $P$ is $\epsilon$-graded if and only if $\rho$ is constant on the set of maximal elements.

See Fig. 1 for an example of a sign-graded poset. Note that if $\epsilon$ is identically equal to 1 , i.e., if $(P, \omega)$ is naturally labeled, then a sign-graded poset with respect to $\epsilon$ is just 
a graded poset. Note also that if $P$ is $\epsilon$-graded then $P$ is also $-\epsilon$-graded, where $-\epsilon$ is defined by $(-\epsilon)(x, y)=-\epsilon(x, y)$. Up to a shift, the order polynomial of a sign-graded labeled poset only depends on the underlying poset:

Theorem 2.2. Let $P$ be $\epsilon$-graded and $\mu$-graded. Then

$$
\Omega\left(P, \epsilon ; t-\frac{r(\epsilon)}{2}\right)=\Omega\left(P, \mu ; t-\frac{r(\mu)}{2}\right) .
$$

Proof. Let $\rho_{\epsilon}$ and $\rho_{\mu}$ denote the rank functions of $(P, \epsilon)$ and $(P, \mu)$ respectively, and let $\mathcal{A}(\epsilon)$ denote the set of $(P, \epsilon)$-partitions. Define a function $\xi: \mathcal{A}(\epsilon) \rightarrow \mathbb{Q}^{P}$ by $\xi \sigma(x)=$ $\sigma(x)+\Delta(x)$, where

$$
\Delta(x)=\frac{r(\epsilon)-\rho_{\epsilon}(x)}{2}-\frac{r(\mu)-\rho_{\mu}(x)}{2} .
$$

Table 1:

\begin{tabular}{|l|l|l|l|l|}
\hline$\epsilon(x, y)$ & $\mu(x, y)$ & $\sigma$ & $\Delta$ & $\xi \sigma$ \\
\hline \hline 1 & 1 & $\sigma(x) \geq \sigma(y)$ & $\Delta(x)=\Delta(y)$ & $\xi \sigma(x) \geq \xi \sigma(y)$ \\
\hline 1 & -1 & $\sigma(x) \geq \sigma(y)$ & $\Delta(x)=\Delta(y)+1$ & $\xi \sigma(x)>\xi \sigma(y)$ \\
\hline-1 & 1 & $\sigma(x)>\sigma(y)$ & $\Delta(x)=\Delta(y)-1$ & $\xi \sigma(x) \geq \xi \sigma(y)$ \\
\hline-1 & -1 & $\sigma(x)>\sigma(y)$ & $\Delta(x)=\Delta(y)$ & $\xi \sigma(x)>\xi \sigma(y)$ \\
\hline
\end{tabular}

The four possible combinations of labelings of a covering-relation $(x, y) \in E$ are given in Table 1.

According to the table $\xi \sigma$ is a $(P, \mu)$-partition provided that $\xi \sigma(x)>0$ for all $x \in P$. But $\xi \sigma$ is order-reversing so it attains its minima on maximal elements and if $z$ is a maximal element we have $\xi \sigma(z)=\sigma(z)$. Hence $\xi: \mathcal{A}(\epsilon) \rightarrow \mathcal{A}(\mu)$. By symmetry we also have a map $\eta: \mathcal{A}(\mu) \rightarrow \mathcal{A}(\epsilon)$ defined by

$$
\eta \sigma(x)=\sigma(x)+\frac{r(\mu)-\rho_{\mu}(x)}{2}-\frac{r(\epsilon)-\rho_{\epsilon}(x)}{2} .
$$

Hence, $\eta=\xi^{-1}$ and $\xi$ is a bijection.

Since $\sigma$ and $\xi \sigma$ are order-reversing they attain their maxima on minimal elements. But if $z$ is a minimal element then $\xi \sigma(z)=\sigma(z)+\frac{r(\epsilon)-r(\mu)}{2}$, which gives

$$
\Omega(P, \mu ; n)=\Omega\left(P, \epsilon ; n+\frac{r(\mu)-r(\epsilon)}{2}\right),
$$

for all nonnegative integers $n$ and the theorem follows.

Theorem 2.3. Let $P$ be $\epsilon$-graded. Then

$$
\Omega(P, \epsilon ; t)=(-1)^{p} \Omega(P, \epsilon ;-t-r(\epsilon)) .
$$


Proof. We have the following reciprocity for order polynomials, see [14]:

$$
\Omega(P,-\epsilon ; t)=(-1)^{p} \Omega(P, \epsilon ;-t) .
$$

Note that $r(-\epsilon)=-r(\epsilon)$, so by Theorem 2.2 we have:

$$
\Omega(P,-\epsilon ; t)=\Omega(P, \epsilon ; t-r(\epsilon)),
$$

which, combined with (2.1), gives the desired result.

Corollary 2.4. Let $P$ be an $\epsilon$-graded poset. Then $W(P, \epsilon ; t)$ is symmetric with center of symmetry $(p-r(\epsilon)-1) / 2$. If $P$ is also $\mu$-graded then

$$
W(P, \mu ; t)=t^{(r(\epsilon)-r(\mu)) / 2} W(P, \epsilon ; t) .
$$

Proof. Suppose that $W(P, \epsilon ; t)=\sum_{i \geq 0} w_{i}(P, \epsilon) t^{i}$. From (1.1) it follows that $\Omega(P, \epsilon ; t)=$ $\sum_{i \geq 0} w_{i}(P, \epsilon)\left(\begin{array}{c}t+p-1-i \\ p\end{array}\right)$. Let $r=r(\epsilon)$. Theorem 2.3 gives:

$$
\begin{aligned}
\Omega(P, \epsilon ; t) & =\sum_{i \geq 0} w_{i}(P, \epsilon)(-1)^{p}\left(\begin{array}{c}
-t-r+p-1-i \\
p
\end{array}\right) \\
& =\sum_{i \geq 0} w_{i}(P, \epsilon)\left(\begin{array}{c}
t+r+i \\
p
\end{array}\right) \\
& =\sum_{i \geq 0} w_{p-r-1-i}(P, \epsilon)\left(\begin{array}{c}
t+p-1-i \\
p
\end{array}\right),
\end{aligned}
$$

so $w_{i}(P, \epsilon)=w_{p-r-1-i}(P, \epsilon)$ for all $i$, and the symmetry follows. The relationship between the $W$-polynomials of $(P, \epsilon)$ and $(P, \mu)$ follows from Theorem 2.2 and the expansion of order-polynomials in the basis $\left(\begin{array}{c}t+p-1-i \\ p\end{array}\right)$.

We say that a poset $P$ is parity graded if the size of all maximal chains in $P$ have the same parity. Also, a poset is $P$ is parity consistent if for all $x \in P$ the order ideal $\Lambda_{x}$ is parity graded. These classes of posets were studied in [12] in a different context. The following theorem tells us that the class of sign-graded posets is considerably greater than the class of graded posets.

Theorem 2.5. Let $P$ be a poset. Then

- there exists a labeling $\epsilon: E \rightarrow\{-1,1\}$ such that $P$ is $\epsilon$-consistent if and only if $P$ is parity consistent,

- there exists a labeling $\epsilon: E \rightarrow\{-1,1\}$ such that $P$ is $\epsilon$-graded if and only if $P$ is parity graded.

Moreover, the labeling $\epsilon$ can be chosen so that the corresponding rank function has values in $\{0,1\}$. 
Proof. It suffices to prove the equivalence regarding parity graded posets. It is clear that if $P$ is $\epsilon$-graded then $P$ is parity graded.

Let $P$ be parity graded. Then, for any $x \in P$, all saturated chains from a minimal element to $x$ have the same length modulo 2. Hence, we may define a labeling $\epsilon: E(P) \rightarrow$ $\{-1,1\}$ by $\epsilon(x, y)=(-1)^{\ell(x)}$, where $\ell(x)$ is the length of any saturated chain starting at a minimal element and ending at $x$. It follows that $P$ is $\epsilon$-graded and that its rank function has values in $\{0,1\}$.

We say that $\omega: P \rightarrow\{1,2, \ldots, p\}$ is canonical if $(P, \omega)$ has a rank-function $\rho$ with values in $\{0,1\}$, and $\rho(x)<\rho(y)$ implies $\omega(x)<\omega(y)$. By Theorem 2.5 we know that $P$ admits a canonical labeling if $P$ is $\epsilon$-consistent for some $\epsilon$.

\section{The Jordan-Hölder set of an $\epsilon$-consistent poset}

Let $P$ be $\omega$-consistent. We may assume that $\omega(x)<\omega(y)$ whenever $\rho(x)<\rho(y)$. This is because any labeling $\omega^{\prime}$ of $P$ for which $\rho(x)<\rho(y)$ implies $\omega^{\prime}(x)<\omega^{\prime}(y)$ will give rise to the same labeling of $E(P)$ as $(P, \omega)$.

Suppose that $x, y \in P$ are incomparable and that $\rho(y)=\rho(x)+1$. Then the JordanHölder set of $(P, \omega)$ can be partitioned into two sets: One where in all permutations $\omega(x)$ comes before $\omega(y)$ and one where $\omega(y)$ always comes before $\omega(x)$. This means that $\mathcal{L}(P, \omega)$ is the disjoint union

$$
\mathcal{L}(P, \omega)=\mathcal{L}\left(P^{\prime}, \omega\right) \sqcup \mathcal{L}\left(P^{\prime \prime}, \omega\right),
$$

where $P^{\prime}$ is the transitive closure of $E \cup\{x \prec y\}$, and $P^{\prime \prime}$ is the transitive closure of $E \cup\{y \prec x\}$.

Lemma 3.1. With definitions as above $P^{\prime}$ and $P^{\prime \prime}$ are $\omega$-consistent with the same rankfunction as $(P, \omega)$.

Proof. Let $c: z_{0} \prec z_{1} \prec \cdots \prec z_{k}=z$ be a saturated chain in $P^{\prime \prime}$, where $z_{0}$ is a minimal element of $P^{\prime \prime}$. Of course $z_{0}$ is also a minimal element of $P$. We have to prove that

$$
\rho(z)=\sum_{i=0}^{k-1} \epsilon^{\prime \prime}\left(z_{i}, z_{i+1}\right),
$$

where $\epsilon^{\prime \prime}$ is the labeling of $E\left(P^{\prime \prime}\right)$ and $\rho$ is the rank-function of $(P, \omega)$.

All covering relations in $P^{\prime \prime}$, except $y \prec x$, are also covering relations in $P$. If $y$ and $x$ do not appear in $c$, then $c$ is a saturated chain in $P$ and there is nothing to prove. Otherwise

$$
c: y_{0} \prec \cdots \prec y_{i}=y \prec x=x_{i+1} \prec x_{i+2} \prec \cdots \prec x_{k}=z .
$$

Note that if $s_{0} \prec s_{1} \prec \cdots \prec s_{\ell}$ is any saturated chain in $P$ then $\sum_{i=0}^{\ell-1} \epsilon\left(s_{i}, s_{i+1}\right)=$ $\rho\left(s_{\ell}\right)-\rho\left(s_{0}\right)$. Since $y_{0} \prec \cdots \prec y_{i}=y$ and $x=x_{i+1} \prec x_{i+2} \prec \cdots \prec x_{k}=z$ are saturated 
chains in $P$ we have

$$
\begin{aligned}
\sum_{i=0}^{k-1} \epsilon^{\prime \prime}\left(z_{i}, z_{i+1}\right) & =\rho(y)+\epsilon^{\prime \prime}(y, x)+\rho(z)-\rho(x) \\
& =\rho(y)-1-\rho(x)+\rho(z) \\
& =\rho(z)
\end{aligned}
$$

as was to be proved. The statement for $\left(P^{\prime}, \omega\right)$ follows similarly.

We say that a $\omega$-consistent poset $P$ is saturated if for all $x, y \in P$ we have that $x$ and $y$ are comparable whenever $|\rho(y)-\rho(x)|=1$. Let $P$ and $Q$ be posets on the same set. Then $Q$ extends $P$ if $x<_{Q} y$ whenever $x<_{P} y$.

Theorem 3.2. Let $P$ be a $\omega$-consistent poset. Then the Jordan-Hölder set of $(P, \omega)$ is uniquely decomposed as the disjoint union

$$
\mathcal{L}(P, \omega)=\bigsqcup_{Q} \mathcal{L}(Q, \omega)
$$

where the union is over all saturated $\omega$-consistent posets $Q$ that extend $P$ and have the same rank-function as $(P, \omega)$.

Proof. That the union exhausts $\mathcal{L}(P, \omega)$ follows from (3.1) and Lemma 3.1. Let $Q_{1}$ and $Q_{2}$ be two different saturated $\omega$-consistent posets that extend $P$ and have the same rankfunction as $(P, \omega)$. We may assume that $Q_{2}$ does not extend $Q_{1}$. Then there exists a covering relation $x \prec y$ in $Q_{1}$ such that $x \nless y$ in $Q_{2}$. Since $|\rho(x)-\rho(y)|=1$ we must have $y<x$ in $Q_{2}$. Thus $\omega(x)$ precedes $\omega(y)$ in any permutation in $\mathcal{L}\left(Q_{1}, \omega\right)$, and $\omega(y)$ precedes $\omega(x)$ in any permutation in $\mathcal{L}\left(Q_{2}, \omega\right)$. Hence, the union is disjoint and unique.

We need two operations on labeled posets: Let $(P, \epsilon)$ and $(Q, \mu)$ be two labeled posets. The ordinal sum, $P \oplus Q$, of $P$ and $Q$ is the poset with the disjoint union of $P$ and $Q$ as underlying set and with partial order defined by $x \leq y$ if $x \leq_{P} y$ or $x \leq_{Q} y$, or $x \in P, y \in Q$. Define two labelings of $E(P \oplus Q)$ by

$$
\begin{aligned}
\left(\epsilon \oplus_{1} \mu\right)(x, y) & =\epsilon(x, y) \text { if }(x, y) \in E(P), \\
\left(\epsilon \oplus_{1} \mu\right)(x, y) & =\mu(x, y) \text { if }(x, y) \in E(Q) \text { and } \\
\left(\epsilon \oplus_{1} \mu\right)(x, y) & =1 \text { otherwise. } \\
\left(\epsilon \oplus_{-1} \mu\right)(x, y) & =\epsilon(x, y) \text { if }(x, y) \in E(P), \\
\left(\epsilon \oplus_{-1} \mu\right)(x, y) & =\mu(x, y) \text { if }(x, y) \in E(Q) \text { and } \\
\left(\epsilon \oplus_{-1} \mu\right)(x, y) & =-1 \text { otherwise. }
\end{aligned}
$$

With a slight abuse of notation we write $P \oplus_{ \pm 1} Q$ when the labelings of $P$ and $Q$ are understood from the context. Note that ordinal sums are associative, i.e., $\left(P \oplus_{ \pm 1} Q\right) \oplus_{ \pm 1}$ $R=P \oplus_{ \pm 1}\left(Q \oplus_{ \pm 1} R\right)$, and preserve the property of being sign-graded. The following result is easily obtained by combinatorial reasoning, see $[2,17]$ : 
Proposition 3.3. Let $(P, \omega)$ and $(Q, \nu)$ be two labeled posets. Then

$$
W\left(P \oplus Q, \omega \oplus_{1} \nu ; t\right)=W(P, \omega ; t) W(Q, \nu ; t)
$$

and

$$
W\left(P \oplus Q, \omega \oplus_{-1} \nu ; t\right)=t W(P, \omega ; t) W(Q, \nu ; t) .
$$

Proposition 3.4. Suppose that $(P, \omega)$ is a saturated canonically labeled $\omega$-consistent poset. Then $(P, \omega)$ is the direct sum

$$
(P, \omega)=A_{0} \oplus_{1} A_{1} \oplus_{-1} A_{2} \oplus_{1} A_{3} \oplus_{-1} \cdots \oplus_{ \pm 1} A_{k},
$$

where the $A_{i}$ s are anti-chains.

Proof. Let $\pi \in \mathcal{L}(P, \omega)$. Then we may write $\pi$ as $\pi=w_{0} w_{1} \cdots w_{k}$ where the $w_{i}$ s are maximal words with respect to the property: If $a$ and $b$ are letters of $w_{i}$ then $\rho\left(\omega^{-1}(a)\right)=$ $\rho\left(\omega^{-1}(b)\right)$. Hence $\pi \in \mathcal{L}(Q, \omega)$ where

$$
(Q, \omega)=A_{0} \oplus_{1} A_{1} \oplus_{-1} A_{2} \oplus_{1} A_{3} \oplus_{-1} \cdots \oplus_{ \pm 1} A_{k},
$$

and $A_{i}$ is the anti-chain consisting of the elements $\omega^{-1}(a)$, where $a$ is a letter of $w_{i}\left(A_{i}\right.$ is an anti-chain, since if $x<y$ where $x, y \in A_{i}$ there would be a letter in $\pi$ between $\omega(x)$ and $\omega(y)$ whose rank was different than that of $x, y)$. Now, $(Q, \omega)$ is saturated so $P=Q$.

Note that the argument in the above proof also can be used to give a simpler proof of Theorem 3.2 when $\omega$ is canonical.

\section{The $W$-polynomial of a sign-graded poset}

The space $S^{d}$ of symmetric polynomials in $\mathbb{R}[t]$ with center of symmetry $d / 2$ has a basis

$$
B_{d}=\left\{t^{i}(1+t)^{d-2 i}\right\}_{i=0}^{\lfloor d / 2\rfloor} .
$$

If $h \in S^{d}$ has nonnegative coefficients in this basis it follows immediately that the coefficients of $h$ in the standard basis are unimodal. Let $S_{+}^{d}$ be the nonnegative span of $B_{d}$. Thus $S_{+}^{d}$ is a cone. Another property of $S_{+}^{d}$ is that if $h \in S_{+}^{d}$ then it has the correct sign at -1 i.e.,

$$
(-1)^{d / 2} h(-1) \geq 0 .
$$

Lemma 4.1. Let $c, d \in \mathbb{N}$. Then

$$
\begin{array}{rll}
S^{c} S^{d} & \subset & S^{c+d} \\
S_{+}^{c} S_{+}^{d} & \subset & S_{+}^{c+d} .
\end{array}
$$

Suppose further that $h \in S^{d}$ has positive leading coefficient and that all zeros of $h$ are real and non-positive. Then $h \in S_{+}^{d}$. 
Proof. The inclusions are obvious. Since $t \in S_{+}^{2}$ and $(1+t) \in S_{+}^{1}$ we may assume that none of them divides $h$. But then we may collect the zeros of $h$ in pairs $\left\{\theta, \theta^{-1}\right\}$. Let $A_{\theta}=-\theta-\theta^{-1}$. Then

$$
h=C \prod_{\theta<-1}\left(t^{2}+A_{\theta} t+1\right)
$$

where $C>0$. Since $A_{\theta}>2$ we have

$$
t^{2}+A_{\theta} t+1=(t+1)^{2}+\left(A_{\theta}-2\right) t \in S_{+}^{2},
$$

and the lemma follows.

We can now prove our main theorem.

Theorem 4.2. Suppose that $(P, \omega)$ is a sign-graded poset of rank $r$. Then $W(P, \omega ; t) \in$ $S_{+}^{p-r-1}$.

Proof. By Corollary 2.4 and Lemma 2.5 we may assume that $(P, \omega)$ is canonically labeled. If $Q$ extends $P$ then the maximal elements of $Q$ are also maximal elements of $P$. By Theorem 3.2 we know that

$$
W(P, \omega ; t)=\sum_{Q} W(Q, \omega ; t)
$$

where $(Q, \omega)$ is saturated and sign-graded with the same rank function and rank as $(P, \omega)$. The $W$-polynomials of anti-chains are the Eulerian polynomials, which have real nonnegative zeros only. By Propositions 3.3 and 3.4 the polynomial $W(Q, \omega ; t)$ has only real non-positive zeros so by Lemma 4.1 and Corollary 2.4 we have $W(Q, \omega ; t) \in S_{+}^{p-r-1}$. The theorem now follows since $S_{+}^{p-r-1}$ is a cone.

Corollary 4.3. Let $(P, \omega)$ be sign-graded of rank $r$. Then $W(P, \omega ; t)$ is symmetric and its coefficients are unimodal. Moreover, $W(P, \omega ; t)$ has the correct sign at -1 , i.e.,

$$
(-1)^{(p-1-r) / 2} W(P, \omega ;-1) \geq 0 .
$$

Corollary 4.4. Let $P$ be a graded poset. Suppose that $\Delta_{e q}(P)$ is flag. Then the CharneyDavis Conjecture holds for $\Delta_{e q}(P)$.

Theorem 4.5. Suppose that $P$ is an $\omega$-consistent poset and that $|\rho(x)-\rho(y)| \leq 1$ for all maximal elements $x, y \in P$. Then $W(P, \omega ; t)$ has unimodal coefficients.

Proof. Suppose that the ranks of maximal elements are contained in $\{r, r+1\}$. If $Q$ is any saturated poset that extends $P$ and has the same rank function as $(P, \omega)$ then $Q$ is $\omega$-graded of rank $r$ or $r+1$. By Theorems 3.2 and 4.2 we know that

$$
W(P, \omega ; t)=\sum_{Q} W(Q, \omega ; t)
$$

where $W(Q, \omega ; t)$ is symmetric and unimodal with center of symmetry at $(p-1-r) / 2$ or $(p-2-r) / 2$. The sum of such polynomials is again unimodal. 


\section{The Charney-Davis quantity}

In [9] Reiner, Stanton and Welker defined the Charney-Davis quantity of a graded naturally labeled poset $(P, \omega)$ of rank $r$ to be

$$
C D(P, \omega)=(-1)^{(p-1-r) / 2} W(P, \omega ;-1) .
$$

We define it in the exact same way for sign-graded posets. Since, by Corollary 2.4, the particular labeling does not matter we write $C D(P)$. Let $\pi=\pi_{1} \pi_{2} \cdots \pi_{n}$ be any permutation. We say that $\pi$ is alternating if $\pi_{1}>\pi_{2}<\pi_{3}>\cdots$ and reverse alternating if $\pi_{1}<\pi_{2}>\pi_{3}<\cdots$. Let $(P, \omega)$ be a canonically labeled sign-graded poset. If $\pi \in \mathcal{L}(P, \omega)$ then we may write $\pi$ as $\pi=w_{0} w_{1} \cdots w_{k}$ where $w_{i}$ are maximal words with respect to the property: If $a$ and $b$ are letters of $w_{i}$ then $\rho\left(\omega^{-1}(a)\right)=\rho\left(\omega^{-1}(b)\right)$. The words $w_{i}$ are called the components of $\pi$. The following theorem is well known, see for example [5, 11, 13], and gives the Charney-Davis quantity of an anti-chain.

Proposition 5.1. Let $n \geq 0$ be an integer. Then $(-1)^{(n-1) / 2} A_{n}(-1)$ is equal to 0 if $n$ is even and equal to the number of (reverse) alternating permutations of the set $\{1,2, \ldots, n\}$ if $n$ is odd.

Theorem 5.2. Let $(P, \omega)$ be a canonically labeled sign-graded poset. Then the CharneyDavis quantity, $C D(P)$, is equal to the number of reverse alternating permutations in $\mathcal{L}(P, \omega)$ such that all components have an odd number of letters.

Proof. It suffices to prove the theorem when $(P, \omega)$ is saturated. By Proposition 3.4 we know that

$$
(P, \omega)=A_{0} \oplus_{1} A_{1} \oplus_{-1} A_{2} \oplus_{1} A_{3} \oplus_{-1} \cdots \oplus_{ \pm 1} A_{k},
$$

where the $A_{i}$ s are anti-chains. Thus $C D(P)=C D\left(A_{0}\right) C D\left(A_{1}\right) \cdots C D\left(A_{k}\right)$. Let $\pi=$ $w_{0} w_{1} \cdots w_{k} \in \mathcal{L}(P, \omega)$ where $w_{i}$ is a permutation of $\omega\left(A_{i}\right)$. Then $\pi$ is a reverse alternating permutation such that all components have an odd number of letters if and only if, for all $i, w_{i}$ is reverse alternating if $i$ is even and alternating if $i$ is odd. Hence, by Proposition 5.1 , the number of such permutations is indeed $C D\left(A_{0}\right) C D\left(A_{1}\right) \cdots C D\left(A_{k}\right)$.

If $h(t)$ is any polynomial with integer coefficients and $h(t) \in S^{d}$, it follows that $h(t)$ has integer coefficients in the basis $t^{i}(1+t)^{d-2 i}$. Thus we know that if $(P, \omega)$ is sign-graded of rank $r$, then

$$
W(P, \omega ; t)=\sum_{i=0}^{\lfloor(p-r-1) / 2\rfloor} a_{i}(P, \omega) t^{i}(1+t)^{p-r-1-2 i},
$$

where $a_{i}(P, \omega)$ are nonnegative integers. By Theorem 5.2 we have a combinatorial interpretation of the $a_{(p-r-1) / 2}(P, \omega)$. A similar but more complicated interpretation of $a_{i}(P, \omega), i=0,1, \ldots,\lfloor(p-r-1) / 2\rfloor$ can be deduced from Proposition 3.4 and the work in $[5,11]$. We omit this. 


\section{The right mode}

Let $f(x)=a_{0}+a_{1} x+\cdots+a_{d} x^{d}$ be a polynomial with real coefficients. The mode, $\operatorname{mode}(f)$, of $f$ is the average value of the indices $i$ such that $a_{i}=\max \left\{a_{j}\right\}_{j=0}^{d}$. One can easily compute the mode of a polynomial with real non-positive zeros only:

Theorem 6.1. [4] Let $f$ be a polynomial with real non-positive zeros only and with positive leading coefficient. Then

$$
\left|\frac{f^{\prime}(1)}{f(1)}-\operatorname{mode}(f)\right|<1 .
$$

It is known, see $[2,14,17]$, that

$$
W(P, \omega ; x)=\sum_{i=1}^{p} e_{i}(P, \omega) x^{i-1}(1-x)^{p-i}
$$

where $e_{i}(P, \omega)$ is the number of surjective $(P, \omega)$-partitions $\sigma: P \rightarrow\{1,2, \ldots, i\}$. A simple calculation gives

$$
\frac{W^{\prime}(P, \omega ; 1)}{W(P, \omega ; 1)}=p-1-\frac{e_{p-1}(P, \omega)}{e_{p}(P, \omega)} .
$$

If $P$ is $\omega$-graded of rank $r$ we know by Theorem 4.2 that mode $(W(P, \omega ; x))=(p-r-1) / 2$. The Neggers-Stanley conjecture, Theorem 6.1 and (6.1) suggest that $2 e_{p-1}(P, \omega)=(p+$ $r-1) e_{p}(P, \omega)$. Stanley [14] proved this for graded posets and it generalizes to sign-graded posets:

Proposition 6.2. Let $P$ be $\omega$-graded of rank $r$. Then

$$
2 e_{p-1}(P, \omega)=(p+r-1) e_{p}(P, \omega) .
$$

Proof. The identity follows when expanding $\Omega(P, \omega ; t)$ in powers of $t$ using Theorem 2.3. See [14, Corollary 19.4] for details.

\section{A characterization of sign-graded posets}

Here we give a characterization of sign-graded posets along the lines of the characterization of graded posets given by Stanley in [14]. Let $(P, \epsilon)$ be a labeled poset. Define a function $\delta=\delta_{\epsilon}: P \rightarrow \mathbb{Z}$ by

$$
\delta(x)=\max \left\{\sum_{i=1}^{\ell} \epsilon\left(x_{i-1}, x_{i}\right)\right\},
$$

where $x=x_{0} \prec x_{1} \prec \cdots \prec x_{\ell}$ is any saturated chain starting at $x$ and ending at a maximal element $x_{\ell}$. Define a map $\Phi=\Phi_{\epsilon}: \mathcal{A}(\epsilon) \rightarrow \mathbb{Z}^{P}$ by

$$
\Phi \sigma=\sigma+\delta
$$


We have

$$
\delta(x) \geq \delta(y)+\epsilon(x, y) .
$$

This means that $\Phi \sigma(x)>\Phi \sigma(y)$ if $\epsilon(x, y)=1$ and $\Phi \sigma(x) \geq \Phi \sigma(y)$ if $\epsilon(x, y)=-1$. Thus $\Phi \sigma$ is a $(P,-\epsilon)$-partition provided that $\Phi \sigma(x)>0$ for all $x \in P$. But $\Phi \sigma$ is order reversing so it attains its minimum at maximal elements and for maximal elements, $z$, we have $\Phi \sigma(z)=\sigma(z)$. This shows that $\Phi: \mathcal{A}(\epsilon) \rightarrow \mathcal{A}(-\epsilon)$ is an injection.

The dual, $\left(P^{*}, \epsilon^{*}\right)$, of a labeled poset $(P, \epsilon)$ is defined by $x<P^{*} y$ if and only if $y<P_{P^{*}} x$, with labeling defined by $\epsilon^{*}(y, x)=-\epsilon(x, y)$. We say that $P$ is dual $\epsilon$-consistent if $P^{*}$ is $\epsilon^{*}$-consistent.

Proposition 7.1. Let $(P, \epsilon)$ be labeled poset. Then $\Phi_{\epsilon}: \mathcal{A}(\epsilon) \rightarrow \mathcal{A}(-\epsilon)$ is a bijection if and only if $P$ is dual $\epsilon$-consistent.

Proof. If $P$ is dual $\epsilon$-consistent then $P$ is also dual $-\epsilon$-consistent and $\delta_{-\epsilon}(x)=-\delta_{\epsilon}(x)$ for all $x \in P$. Thus the if part follows since the inverse of $\Phi_{\epsilon}$ is $\Phi_{-\epsilon}$.

For the only if direction note that $P$ is dual $\epsilon$-consistent if and only if for all $(x, y) \in E$ we have

$$
\delta(x)=\delta(y)+\epsilon(x, y)
$$

Hence, if $P$ is not dual $\epsilon$-consistent then by (7.1), there is a covering relation $\left(x_{0}, y_{0}\right) \in E$ such that either $\epsilon\left(x_{0}, y_{0}\right)=1$ and $\delta\left(x_{0}\right) \geq \delta\left(y_{0}\right)+2$ or $\epsilon\left(x_{0}, y_{0}\right)=-1$ and $\delta\left(x_{0}\right) \geq \delta\left(y_{0}\right)$.

Suppose that $\epsilon\left(x_{0}, y_{0}\right)=1$. It is clear that there is a $\sigma \in \mathcal{A}(-\epsilon)$ such that $\sigma\left(x_{0}\right)=$ $\sigma\left(y_{0}\right)+1$. But then

$$
\sigma\left(x_{0}\right)-\delta\left(x_{0}\right) \leq \sigma\left(y_{0}\right)-\delta\left(y_{0}\right)-1,
$$

so $\sigma-\delta \notin \mathcal{A}(\epsilon)$.

Similarly, if $\epsilon\left(x_{0}, y_{0}\right)=-1$ then we can find a partition $\sigma \in \mathcal{A}(-\epsilon)$ with $\sigma\left(x_{0}\right)=\sigma\left(y_{0}\right)$, and then

so $\sigma-\delta \notin \mathcal{A}(\epsilon)$.

$$
\sigma\left(x_{0}\right)-\delta\left(x_{0}\right) \leq \sigma\left(y_{0}\right)-\delta\left(y_{0}\right),
$$

Let $(P, \epsilon)$ be a labeled poset. Define $r(\epsilon)$ by

$$
r(\epsilon)=\max \left\{\sum_{i=1}^{\ell} \epsilon\left(x_{i-1}, x_{i}\right): x_{0} \prec x_{1} \prec \cdots \prec x_{\ell} \text { is maximal }\right\} .
$$

We then have:

$$
\begin{aligned}
\max \{\Phi \sigma(x): x \in P\} & =\max \left\{\sigma(x)+\delta_{\epsilon}(x): x \text { is minimal }\right\} \\
& \leq \max \{\sigma(x): x \in P\}+r(\epsilon) .
\end{aligned}
$$

So if we let $\mathcal{A}_{n}(\epsilon)$ be the $(P, \epsilon)$-partitions with largest part at most $n$ we have that $\Phi_{\epsilon}: \mathcal{A}_{n}(\epsilon) \rightarrow \mathcal{A}_{n+r(\epsilon)}(-\epsilon)$ is an injection. A labeling $\epsilon$ of $P$ is said to satisfy the $\lambda$-chain condition if for every $x \in P$ there is a maximal chain $c: x_{0} \prec x_{1} \prec \cdots \prec x_{\ell}$ containing $x$ such that $\sum_{i=1}^{\ell} \epsilon\left(x_{i-1}, x_{i}\right)=r(\epsilon)$. 
Lemma 7.2. Suppose that $n$ is a nonnegative integer such that $\Omega(P, \epsilon ; n) \neq 0$. If

$$
\Omega(P,-\epsilon ; n+r(\epsilon))=\Omega(P, \epsilon ; n)
$$

then $\epsilon$ satisfies the $\lambda$-chain condition.

Proof. Define $\delta^{*}: P \rightarrow \mathbb{Z}$ by

$$
\delta^{*}(x)=\max \left\{\sum_{i=1}^{\ell} \epsilon\left(x_{i-1}, x_{i}\right): x_{0} \prec x_{1} \prec \cdots \prec x_{\ell}=x\right\},
$$

where the maximum is taken over all maximal chains starting at a minimal element and ending at $x$. Then

$$
\delta(x)+\delta^{*}(x) \leq r(\epsilon)
$$

for all $x$, and $\epsilon$ satisfies the $\lambda$-chain condition if and only if we have equality in (7.2) for all $x \in P$. It is easy to see that the map $\Phi^{*}: \mathcal{A}_{n}(\epsilon) \rightarrow \mathcal{A}_{n+r(\epsilon)}(-\epsilon)$ defined by

$$
\Phi^{*} \sigma(x)=\sigma(x)+r(\epsilon)-\delta^{*}(x),
$$

is well-defined and is an injection. By (7.2) we have $\Phi \sigma(x) \leq \Phi^{*} \sigma(x)$ for all $\sigma$ and all $x \in P$, with equality if and only if $x$ is in a maximal chain of maximal weight. This means that in order for $\Phi: \mathcal{A}_{n}(\epsilon) \rightarrow \mathcal{A}_{n+r(\epsilon)}(-\epsilon)$ to be a bijection it is necessary for $\epsilon$ to satisfy the $\lambda$-chain condition.

Theorem 7.3. Let $\epsilon$ be a labeling of $P$. Then

$$
\Omega(P, \epsilon ; t)=(-1)^{p} \Omega(P, \epsilon ;-t-r(\epsilon))
$$

if and only if $P$ is $\epsilon$-graded of rank $r(\epsilon)$.

Proof. The "if" part is Theorem 2.3, so suppose that the equality of the theorem holds. By reciprocity we have

$$
(-1)^{p} \Omega(P, \epsilon ;-t-r(\epsilon))=\Omega(P,-\epsilon ; t+r(\epsilon)),
$$

and since $\Phi_{\epsilon}: \mathcal{A}_{n}(\epsilon) \rightarrow \mathcal{A}_{n+r(\epsilon)}(-\epsilon)$ is an injection it is also a bijection. By Proposition 7.1 we have that $P$ is dual $\epsilon$-consistent and by Lemma 7.2, we have that all minimal elements are members of maximal chains of maximal weight. In other words $P$ is $\epsilon$-graded.

It should be noted that it is not necessary for $P$ to be $\epsilon$-graded in order for $W(P, \epsilon ; t)$ to be symmetric. For example, if $(P, \epsilon)$ is any labeled poset then the $W$-polynomial of the disjoint union of $(P, \epsilon)$ and $(P,-\epsilon)$ is easily seen to be symmetric. However, we have the following:

Corollary 7.4. Suppose that

$$
\Omega(P, \epsilon ; t)=\Omega(P,-\epsilon ; t+s),
$$

for some $s \in \mathbb{Z}$. Then $-r(-\epsilon) \leq s \leq r(\epsilon)$, with equality if and only if $P$ is $\epsilon$-graded.

Proof. We have an injection $\Phi_{\epsilon}: \mathcal{A}_{n}(\epsilon) \rightarrow \mathcal{A}_{n+r(\epsilon)}(-\epsilon)$. This means that $s \leq r(\epsilon)$. The lower bound follows from the injection $\Phi_{-\epsilon}$, and the statement of equality follows from Theorem 7.3. 


\section{References}

[1] P. Brändén, On operators on polynomials preserving real-rootedness and the NeggersStanley Conjecture, J.Algebraic Comb. (to appear).

[2] F. Brenti, Unimodal, log-concave and Pólya frequency sequences in combinatorics, Mem. Amer. Math. Soc. 81 (1989).

[3] R. Charney and M. Davis, The Euler characteristic of a nonpositively curved, piecewise Euclidean manifold, Pacific J. Math. 171 (1995), 117-137.

[4] J. N. Darroch, On the distribution of the number of successes in independent trials, Ann. Math. Statist. 35 (1965), 1317-1321.

[5] D. Foata and V. Strehl, Euler numbers and variations of permutations, in Colloquio Internazionale sulle Teorie Combinatorie 1973, Tome I (Atti Dei Convegni Lincei, 17, 119-131), Accademia Nazionale dei Lincei, 1976.

[6] S. Gal, Real Root Conjecture fails for five and higher dimensional spheres, Preprint.

[7] V. Gasharov, On the Neggers-Stanley conjecture and the Eulerian polynomials, J. Combin. Theory Ser. A 82 (1998), 134-146.

[8] J. Neggers, Representations of finite partially ordered sets, J. Combin. Inform. System Sci 3 (1978), 113-133.

[9] V. Reiner and D. Stanton and V. Welker, The Charney-Davis Quantity for certain graded posets, Sém. Lothar. Combin. 50 (2003).

[10] V. Reiner and V. Welker, On the Charney-Davis and the Neggers-Stanley Conjectures, J. Combin. Theory Ser. A (to appear).

[11] L. W. Shapiro and W. J. Jin and S. Seyoum, Runs, slides and moments, SIAM J. Algebraic Discrete Methods 4 (1983), 459-466.

[12] R. P. Stanley, Some remarks on sign-balanced and maj-balanced posets, Advances in Applied Math. (to appear).

[13] R. P. Stanley, Enumerative combinatorics. Vol. 1, Cambridge University Press (1997).

[14] R. P. Stanley, Ordered structures and partitions, Mem. Amer. Math. Soc. 119 (1972).

[15] R. P. Stanley, The number of faces of simplicial polytopes and spheres. In Discrete geometry and convexity, New York Acad. Sci. (1985).

[16] R. P. Stanley, Combinatorics and commutative algebra, Birkhäuser Boston Inc. (1996).

[17] D. G. Wagner, Enumeration of functions from posets to chains, European J. Combin. 13 (1992). 